\title{
Solution to isotope amount ratio measurement challenge
}

\author{
Olaf Rienitz $^{1} \cdot$ Axel Pramann ${ }^{1}$
}

(C) Springer-Verlag Berlin Heidelberg 2016

\section{Solution}

The isotope ratio in mixture $\mathrm{AB}$ is the key to the answer posed in this Challenge [1]. Therefore, let us start with its isotope amount ratio which can be expressed as the sum of the amounts of ${ }^{65} \mathrm{Cu}$ and ${ }^{63} \mathrm{Cu}$ originating from bottles $\mathrm{A}$ and $\mathrm{B}$, respectively:

$R_{\mathrm{AB}}=\frac{n_{\mathrm{AB}}\left({ }^{65} \mathrm{Cu}\right)}{n_{\mathrm{AB}}\left({ }^{63} \mathrm{Cu}\right)}=\frac{n_{\mathrm{A}}\left({ }^{65} \mathrm{Cu}\right)+n_{\mathrm{B}}\left({ }^{65} \mathrm{Cu}\right)}{n_{\mathrm{A}}\left({ }^{63} \mathrm{Cu}\right)+n_{\mathrm{B}}\left({ }^{63} \mathrm{Cu}\right)}$

For example, the amount of ${ }^{65} \mathrm{Cu}$ in mixture $\mathrm{AB}$ originating from bottle $\mathrm{A}\left(n_{\mathrm{A}}\left({ }^{65} \mathrm{Cu}\right)\right)$ can be expressed with the amount fraction of ${ }^{65} \mathrm{Cu}$ in bottle $\mathrm{A}\left(x_{\mathrm{A}}\left({ }^{65} \mathrm{Cu}\right)\right)$ and the total amount of substance in mixture $\mathrm{AB}$ from bottle $\mathrm{A}\left(n_{\mathrm{A}}\right)$ :

$n_{\mathrm{A}}\left({ }^{65} \mathrm{Cu}\right)=x_{\mathrm{A}}\left({ }^{65} \mathrm{Cu}\right) \times n_{\mathrm{A}}$

The total amount of copper in mixture AB from bottle A $\left(n_{\mathrm{A}}\right)$ can be rewritten using the molar mass of copper in bottle $\mathrm{A}\left(M_{\mathrm{A}}\right)$ and the total mass of copper in mixture $\mathrm{AB}$ from bottle $\mathrm{A}\left(m_{\mathrm{A}}(\mathrm{Cu})\right)$ :

$n_{\mathrm{A}}=\frac{m_{\mathrm{A}}(\mathrm{Cu})}{M_{\mathrm{A}}}$

This article is the solution to the Analytical Challenge to be found at http://dx.doi.org/10.1007/s00216-016-9744-0.

\section{Olaf Rienitz}

olaf.rienitz@ptb.de

1 Physikalisch-Technische Bundesanstalt, Bundesallee 100, 38116 Braunschweig, Germany
Combining Eqs. (1)-(3) yields

$$
\begin{aligned}
R_{\mathrm{AB}}= & \frac{x_{\mathrm{A}}\left({ }^{65} \mathrm{Cu}\right) \times n_{\mathrm{A}}+x_{\mathrm{B}}\left({ }^{65} \mathrm{Cu}\right) \times n_{\mathrm{B}}}{x_{\mathrm{A}}\left({ }^{63} \mathrm{Cu}\right) \times n_{\mathrm{A}}+x_{\mathrm{B}}\left({ }^{63} \mathrm{Cu}\right) \times n_{\mathrm{B}}} \\
= & \frac{x_{\mathrm{A}}\left({ }^{65} \mathrm{Cu}\right) \times \frac{m_{\mathrm{A}}(\mathrm{Cu})}{M_{\mathrm{A}}}+x_{\mathrm{B}}\left({ }^{65} \mathrm{Cu}\right) \times \frac{m_{\mathrm{B}}(\mathrm{Cu})}{M_{\mathrm{B}}}}{x_{\mathrm{A}}\left({ }^{63} \mathrm{Cu}\right) \times \frac{m_{\mathrm{A}}(\mathrm{Cu})}{M_{\mathrm{A}}}+x_{\mathrm{B}}\left({ }^{63} \mathrm{Cu}\right) \times \frac{m_{\mathrm{B}}(\mathrm{Cu})}{M_{\mathrm{B}}}}
\end{aligned}
$$

The relation between the isotope ratios and the amount fractions

$x_{i}\left({ }^{63} \mathrm{Cu}\right)=\frac{1}{1+R_{i}} \quad x_{i}\left({ }^{65} \mathrm{Cu}\right)=\frac{R_{i}}{1+R_{i}}$

helps to eliminate the amount fractions from Eq. (4):

$$
R_{\mathrm{AB}}=\frac{\frac{R_{\mathrm{A}}}{1+R_{\mathrm{A}}} \times \frac{m_{\mathrm{A}}(\mathrm{Cu})}{M_{\mathrm{A}}}+\frac{R_{\mathrm{B}}}{1+R_{\mathrm{B}}} \times \frac{m_{\mathrm{B}}(\mathrm{Cu})}{M_{\mathrm{B}}}}{\frac{1}{1+R_{\mathrm{A}}} \times \frac{m_{\mathrm{A}}(\mathrm{Cu})}{M_{\mathrm{A}}}+\frac{1}{1+R_{\mathrm{B}}} \times \frac{m_{\mathrm{B}}(\mathrm{Cu})}{M_{\mathrm{B}}}}
$$

But the molar masses of the copper in bottles A and B are still unknown. They can be rewritten using the amount fractions and the corresponding molar masses of the isotopes (e.g., bottle A):

$M_{\mathrm{A}}=x_{\mathrm{A}}\left({ }^{63} \mathrm{Cu}\right) \times M\left(\left({ }^{63} \mathrm{Cu}\right)+x_{\mathrm{A}}\left({ }^{65} \mathrm{Cu}\right) \times M\left({ }^{65} \mathrm{Cu}\right)\right.$

Like before, we can eliminate the amount fractions using Eq. (5):

$$
\begin{aligned}
M_{\mathrm{A}} & =\frac{1}{1+R_{\mathrm{A}}} \times M\left({ }^{63} \mathrm{Cu}\right)+\frac{R_{\mathrm{A}}}{1+R_{\mathrm{A}}} \times M\left({ }^{65} \mathrm{Cu}\right) \\
& =\frac{M\left({ }^{63} \mathrm{Cu}\right)+R_{\mathrm{A}} \times M\left({ }^{65} \mathrm{Cu}\right)}{1+R_{\mathrm{A}}}
\end{aligned}
$$


Thus, Eq. (6) becomes

$$
R_{\mathrm{AB}}=\frac{\frac{R_{\mathrm{A}}}{1+R_{\mathrm{A}}} \times \frac{m_{\mathrm{A}}(\mathrm{Cu})}{\frac{M\left({ }^{63} \mathrm{Cu}\right)+R_{\mathrm{A}} \times M\left({ }^{65} \mathrm{Cu}\right)}{1+R_{\mathrm{A}}}+\frac{R_{\mathrm{B}}}{1+R_{\mathrm{B}}} \times \frac{m_{\mathrm{B}}(\mathrm{Cu})}{\frac{M\left({ }^{63} \mathrm{Cu}\right)+R_{\mathrm{B}} \times M\left({ }^{65} \mathrm{Cu}\right)}{1+R_{\mathrm{B}}}}}}{\frac{m_{\mathrm{A}}(\mathrm{Cu})}{1+R_{\mathrm{A}}} \times \frac{m_{\mathrm{B}}(\mathrm{Cu})}{\frac{M\left({ }^{63} \mathrm{Cu}\right)+R_{\mathrm{A}} \times M\left({ }^{65} \mathrm{Cu}\right)}{1+R_{\mathrm{A}}}}+\frac{1}{1+R_{\mathrm{B}}} \times \frac{M\left({ }^{63} \mathrm{Cu}\right)+R_{\mathrm{B}} \times M\left({ }^{65} \mathrm{Cu}\right)}{1+R_{\mathrm{B}}}}
$$

The terms $\left(1+R_{\mathrm{A}}\right)$ and $\left(1+R_{\mathrm{B}}\right)$ can be canceled and Eq. (9) yields

$R_{\mathrm{AB}}=\frac{\frac{R_{\mathrm{A}} \times m_{\mathrm{A}}(\mathrm{Cu})}{M\left({ }^{63} \mathrm{Cu}\right)+R_{\mathrm{A}} \times M\left({ }^{65} \mathrm{Cu}\right)}+\frac{R_{\mathrm{B}} \times m_{\mathrm{B}}(\mathrm{Cu})}{M\left({ }^{63} \mathrm{Cu}\right)+R_{\mathrm{B}} \times M\left({ }^{65} \mathrm{Cu}\right)}}{\frac{m_{\mathrm{A}}(\mathrm{Cu})}{M\left({ }^{63} \mathrm{Cu}\right)+R_{\mathrm{A}} \times M\left({ }^{65} \mathrm{Cu}\right)}+\frac{m_{\mathrm{B}}(\mathrm{Cu})}{M\left({ }^{63} \mathrm{Cu}\right)+R_{\mathrm{B}} \times M\left({ }^{65} \mathrm{Cu}\right)}}$

An expression that merely contains known and measured quantities except for the desired calibration factor $(K)$ is obtained after replacing the isotope amount ratios $\left(R_{i}\right)$ with the signal intensity ratios $\left(r_{i}\right)$ and the calibration factor $(K)$ :

$$
\begin{aligned}
& K \times r_{\mathrm{AB}}= \\
& \frac{\frac{K \times r_{\mathrm{A}} \times m_{\mathrm{A}}(\mathrm{Cu})}{M\left({ }^{63} \mathrm{Cu}\right)+K \times r_{\mathrm{A}} \times M\left({ }^{65} \mathrm{Cu}\right)}+\frac{K \times r_{\mathrm{B}} \times m_{\mathrm{B}}(\mathrm{Cu})}{M\left({ }^{63} \mathrm{Cu}\right)+K \times r_{\mathrm{B}} \times M\left({ }^{65} \mathrm{Cu}\right)}}{\frac{m_{\mathrm{B}}(\mathrm{Cu})}{M\left({ }^{63} \mathrm{Cu}\right)+K \times r_{\mathrm{A}} \times M\left({ }^{65} \mathrm{Cu}\right)}+\frac{1}{M\left({ }^{63} \mathrm{Cu}\right)+K \times r_{\mathrm{B}} \times M\left({ }^{65} \mathrm{Cu}\right)}}
\end{aligned}
$$

Dividing Eq. (11) by $K$ and multiplying with the denominator yields

$$
\begin{aligned}
& \frac{r_{\mathrm{AB}} \times m_{\mathrm{A}}(\mathrm{Cu})}{M\left({ }^{63} \mathrm{Cu}\right)+K \times r_{\mathrm{A}} \times M\left({ }^{65} \mathrm{Cu}\right)}+ \\
& \frac{r_{\mathrm{AB}} \times m_{\mathrm{B}}(\mathrm{Cu})}{M\left({ }^{63} \mathrm{Cu}\right)+K \times r_{\mathrm{B}} \times M\left({ }^{65} \mathrm{Cu}\right)} \\
& =\frac{r_{\mathrm{A}} \times m_{\mathrm{A}}(\mathrm{Cu})}{M\left({ }^{63} \mathrm{Cu}\right)+K \times r_{\mathrm{A}} \times M\left({ }^{65} \mathrm{Cu}\right)}+ \\
& \frac{r_{\mathrm{B}} \times m_{\mathrm{B}}(\mathrm{Cu})}{M\left({ }^{63} \mathrm{Cu}\right)+K \times r_{\mathrm{B}} \times M\left({ }^{65} \mathrm{Cu}\right)}
\end{aligned}
$$

This equation can now be rearranged to solve for the calibration factor $(K)$ :

$$
\begin{gathered}
{\left[M\left({ }^{63} \mathrm{Cu}\right)+K r_{\mathrm{B}} M\left({ }^{65} \mathrm{Cu}\right)\right] r_{\mathrm{AB}} m_{\mathrm{A}}(\mathrm{Cu})+} \\
\frac{\left[M\left({ }^{63} \mathrm{Cu}\right)+K r_{\mathrm{A}} M\left({ }^{65} \mathrm{Cu}\right)\right] r_{\mathrm{AB}} m_{\mathrm{B}}(\mathrm{Cu})}{\left[M\left({ }^{63} \mathrm{Cu}\right)+K r_{\mathrm{A}} M\left({ }^{65} \mathrm{Cu}\right)\right]\left[M\left({ }^{63} \mathrm{Cu}\right)+K r_{\mathrm{B}} M\left({ }^{65} \mathrm{Cu}\right)\right]} \\
=\frac{\left[M\left({ }^{63} \mathrm{Cu}\right)+K r_{\mathrm{B}} M\left({ }^{65} \mathrm{Cu}\right)\right] r_{\mathrm{A}} m_{\mathrm{A}}(\mathrm{Cu})+}{\left[M\left({ }^{63} \mathrm{Cu}\right)+K r_{\mathrm{A}} M\left({ }^{65} \mathrm{Cu}\right)\right] r_{\mathrm{B}} m_{\mathrm{B}}(\mathrm{Cu})} \\
{\left[M\left({ }^{63} \mathrm{Cu}\right)+K r_{\mathrm{A}} M\left({ }^{65} \mathrm{Cu}\right)\right]\left[M\left({ }^{63} \mathrm{Cu}\right)+K r_{\mathrm{B}} M\left({ }^{65} \mathrm{Cu}\right)\right]}
\end{gathered}
$$

After eliminating the common denominator, we obtain

$$
\begin{aligned}
& r_{\mathrm{AB}} m_{\mathrm{A}}(\mathrm{Cu}) M\left({ }^{63} \mathrm{Cu}\right)+K r_{\mathrm{B}} r_{\mathrm{AB}} m_{\mathrm{A}}(\mathrm{Cu}) M\left({ }^{65} \mathrm{Cu}\right)+ \\
& r_{\mathrm{AB}} m_{\mathrm{B}}(\mathrm{Cu}) M\left({ }^{63} \mathrm{Cu}\right)+K r_{\mathrm{A}} r_{\mathrm{AB}} m_{\mathrm{B}}(\mathrm{Cu}) M\left({ }^{65} \mathrm{Cu}\right) \\
& =r_{\mathrm{A}} m_{\mathrm{A}}(\mathrm{Cu}) M\left({ }^{63} \mathrm{Cu}\right)+K r_{\mathrm{A}} r_{\mathrm{B}} m_{\mathrm{A}}(\mathrm{Cu}) M\left({ }^{65} \mathrm{Cu}\right)+ \\
& r_{\mathrm{B}} m_{\mathrm{B}}(\mathrm{Cu}) M\left({ }^{63} \mathrm{Cu}\right)+K r_{\mathrm{A}} r_{\mathrm{B}} m_{\mathrm{B}}(\mathrm{Cu}) M\left({ }^{65} \mathrm{Cu}\right)
\end{aligned}
$$

From here,

$$
\begin{aligned}
& K \times M\left({ }^{65} \mathrm{Cu}\right)\left[r_{\mathrm{A}} r_{\mathrm{AB}} m_{\mathrm{B}}(\mathrm{Cu})+r_{\mathrm{B}} r_{\mathrm{AB}} m_{\mathrm{A}}(\mathrm{Cu})-r_{\mathrm{A}} r_{\mathrm{B}} m_{\mathrm{A}}(\mathrm{Cu})-r_{\mathrm{A}} r_{\mathrm{B}} m_{\mathrm{B}}(\mathrm{Cu})\right] \\
& =M\left({ }^{63} \mathrm{Cu}\right)\left[r_{\mathrm{A}} m_{\mathrm{A}}(\mathrm{Cu})+r_{\mathrm{B}} m_{\mathrm{B}}(\mathrm{Cu})-r_{\mathrm{AB}} m_{\mathrm{A}}(\mathrm{Cu})-r_{\mathrm{AB}} m_{\mathrm{B}}(\mathrm{Cu})\right]
\end{aligned}
$$

and

$$
\begin{aligned}
K= & \frac{M\left({ }^{63} \mathrm{Cu}\right)}{M\left({ }^{65} \mathrm{Cu}\right)} \\
& \times \frac{m_{\mathrm{A}}(\mathrm{Cu}) \times\left(r_{\mathrm{A}}-r_{\mathrm{AB}}\right)+m_{\mathrm{B}}(\mathrm{Cu}) \times\left(r_{\mathrm{B}}-r_{\mathrm{AB}}\right)}{r_{\mathrm{B}} m_{\mathrm{A}}(\mathrm{Cu}) \times\left(r_{\mathrm{AB}}-r_{\mathrm{A}}\right)+r_{\mathrm{A}} m_{\mathrm{B}}(\mathrm{Cu}) \times\left(r_{\mathrm{AB}}-r_{\mathrm{B}}\right)}
\end{aligned}
$$

Since you did not mix solid pieces of copper but rather aqueous solutions with copper mass fractions $w_{\mathrm{A}}$ and $w_{\mathrm{B}}$, the masses of solid copper $\left(m_{\mathrm{A}}(\mathrm{Cu})\right.$ and $\left.m_{\mathrm{B}}(\mathrm{Cu})\right)$ have to be replaced with masses of these solutions $\left(m_{\mathrm{A}}\right.$ and $\left.m_{\mathrm{B}}\right)$ : 


$$
\begin{aligned}
K= & \frac{M\left({ }^{63} \mathrm{Cu}\right)}{M\left({ }^{65} \mathrm{Cu}\right)} \\
& \times \frac{m_{\mathrm{A}} w_{\mathrm{A}} \times\left(r_{\mathrm{A}}-r_{\mathrm{AB}}\right)+m_{\mathrm{B}} w_{\mathrm{B}} \times\left(r_{\mathrm{B}}-r_{\mathrm{AB}}\right)}{m_{\mathrm{A}} w_{\mathrm{A}} r_{\mathrm{B}} \times\left(r_{\mathrm{AB}}-r_{\mathrm{A}}\right)+m_{\mathrm{B}} w_{\mathrm{B}} r_{\mathrm{A}} \times\left(r_{\mathrm{AB}}-r_{\mathrm{B}}\right)}
\end{aligned}
$$

This is the required analytical solution for the calibration factor $K$. Now let us put the given numerical values into Eqs. (17):

$$
\begin{aligned}
K= & \frac{62.929598}{64.927790} \\
& 2.2561 \times 851.63 \times(0.00370125-0.985414)+ \\
\times & \frac{2.6432 \times 725.47 \times(48.6537-0.985414)}{2.2561 \times 851.63 \times 48.6537 \times(0.985414-0.00370125)+} \\
& 2.6432 \times 725.47 \times 0.00370125 \times(0.985414-48.6537) \\
K= & 0.94895(\mathrm{~mol} / \mathrm{mol}) /(\mathrm{V} / \mathrm{V})
\end{aligned}
$$

With this calibration factor, the isotope ratios can be calculated from the signal intensity ratios

$$
\begin{aligned}
R_{i} & =\frac{n_{i}\left({ }^{65} \mathrm{Cu}\right)}{n_{i}\left({ }^{63} \mathrm{Cu}\right)}=K \times r_{i} \\
R_{\mathrm{A}} & =K \times r_{\mathrm{A}}=0.94895 \times 0.00370125 \mathrm{~mol} / \mathrm{mol} \\
& =0.0035123 \mathrm{~mol} / \mathrm{mol}
\end{aligned}
$$

$$
\begin{aligned}
R_{\mathrm{B}} & =K \times r_{\mathrm{B}}=0.94895 \times 48.6537 \mathrm{~mol} / \mathrm{mol} \\
& =46.170 \mathrm{~mol} / \mathrm{mol} \\
R_{\mathrm{AB}} & =K \times r_{\mathrm{AB}}=0.94895 \times 0.985414 \mathrm{~mol} / \mathrm{mol} \\
& =0.93511 \mathrm{~mol} / \mathrm{mol}
\end{aligned}
$$

The isotopic abundances are obtained from these isotope amount ratios using Eq. (5):

\begin{tabular}{lll}
\hline & Bottle A & Bottle B \\
$x\left({ }^{63} \mathrm{Cu}\right)$ in $\mathrm{mol} / \mathrm{mol}$ & 0.9965 & 0.0212 \\
$x\left({ }^{65} \mathrm{Cu}\right)$ in $\mathrm{mol} / \mathrm{mol}$ & 0.0035 & 0.9788 \\
\hline
\end{tabular}

Therefore, you were able to verify that the solutions in bottles A and B are spike solutions enriched in copper-63 and copper-65, respectively. The isotopic enrichment is even larger than what was written on the bottles. Your boss should be happy!

\section{References}

1. Rienitz O, Pramann A. Isotope amount ratio measurement challenge. Anal Bioanal Chem. 2016;408:5953-5. 